http://dx.doi.org/10.18778/2196-8403.2015.07

\author{
JAROSŁAW APTACY
}

\title{
Zwischen Norm und Verwendung. Zum Kasus- gebrauch im Deutschen und Polnischen
}

Celem artykułu jest analiza wypadków niezgodnego z obowiązującą normą językową użycia kategorii przypadka gramatycznego w języku niemieckim i polskim. W odniesieniu do języka niemieckiego, na podstawie autentycznych przykładów, zbadano wypowiedzenia, w których realizowana jest nienormatywna rekcja czasownikowa i przyimkowa. Z analizy wynika, że u użytkowników niemczyzny panuje znaczna niepewność w użyciu przypadka (np. celownik zamiast dopełniacza, mianownik zamiast biernika, dopełniacz zamiast celownika), co w przyszłości może spowodować trwałe zmiany językowe. W języku polskim natomiast stwierdzono wyraźną tendencję do zastępowania dopełniacza, zwłaszcza po negacji zdaniowej, biernikiem.

In dem vorliegenden Beitrag wird der Versuch unternommen, Diskrepanzen zwischen der Norm und Verwendung der Kasuskategorie im Deutschen und Polnischen aufzuzeigen und analytisch zu beschreiben. Bezüglich des Deutschen werden Fälle der adverbalen und adpräpositionalen Kasusrektion anhand authentischer Belege untersucht. Aus der Analyse folgt, dass im Kasusgebrauch eine beachtliche Verunsicherung (z. B. Dativ statt Genitiv, Nominativ statt Akkusativ, Genitiv statt Dativ) zu verzeichnen ist, die künftig zu einem nachhaltigen Sprachwandel führen könnte. Im Polnischen dagegen wird eine allgemeine Tendenz zur Ersetzung des Genitivs durch den Akkusativ, v.a. bei der Satznegation, beobachtet.

The aim of this article is to analyze the use of the grammatical case category in German and Polish in a manner inconsistent with the binding norm. With regard to German, based on real-life examples, utterances have been examined in which the non-normative use of the verbal and prepositional case has been found. The analysis shows that there is a considerable uncertainty in the use of the case among the speakers of German (the dative instead of the genitive, the nominative instead of the accusative, the genitive 
instead of the dative), which in future may cause permanent changes in the language. In Polish, a clear tendency has been observed to replace the genitive with the accusative, especially after a sentential negation.

\section{Allgemeines}

Eine unmittelbare Motivation für die Entstehung dieses Beitrags bilden rezente Sprachverwendungsfälle im Deutschen und im Polnischen, in denen eine Diskrepanz im Kasusgebrauch zwischen der präskriptiven Norm und den tatsächlich geäußerten - mündlichen oder schriftlichen - Texten festzustellen ist. Das Ziel der Analyse besteht in einer exemplarischen Darstellung und Besprechung normwidriger Kasusverwendungen im Deutschen und Polnischen mit Hinweisen auf einen möglichen Sprachwandel, der durch eine solche Handhabe der Kasuskategorie ausgelöst werden könnte. Dabei werden hier als Norm für Deutsch die Angaben in der Duden-Grammatik (2009) sowie in Band 9 der Duden-Serie Richtiges und gutes Deutsch (2007) zugrunde gelegt. Für das Polnische gelten entsprechend das normative Wörterbuch von MARKOWSKI (2002) sowie die normativ ausgerichtete Arbeit von JADACKA (2008). Die deutschen Belege stammen größtenteils aus den Online- und Print-Ausgaben von Frankfurter Allgemeine Zeitung, Die Zeit und Süddeutsche Zeitung. Sollten die Beispiele anderen Quellen entnommen worden sein, so wird dies jeweils an entsprechender Stelle vermerkt. Polnische Belege wurden meist in Bezug auf den Kasusgebrauch in normwidrigen Äußerungen von Politikern und Journalisten entnommen, die in Rundfunk und Fernsehen neuerdings zuhauf zu verzeichnen sind. Die Quellen werden selbstredend auch hier bei jedem Beleg genannt. Die Korpora weisen demnach eine gewisse Asymmetrie auf, die jedoch dadurch begründet werden kann, dass die analysierte Erscheinung im Falle des Deutschen in schriftlich veröffentlichen Quellen belegt werden kann, während sie sich im Polnischen v.a. aufs Mündliche beschränkt und in geschriebenen Texten nur sporadisch auftritt.

\section{Kasus und Rektion}

Kasus wird unter struktureller Beziehung der Rektion zugewiesen. ${ }^{1}$ Als Rektion gilt gemeinhin eine „,[l]exemspezifische Eigenschaft von Verben, Adjektiven, Präpositionen oder Substantiven, die die morphologische Kategorie (ins-

1 Ob der Kasus zugewiesen oder - den neueren syntaktischen Ansätzen im Rahmen des Minimalistischen Programms entsprechend - überprüft wird, ist in diesem 
besondere den Kasus) abhängiger Elemente bestimmt. R[ektion] kann unter [...] Valenz subsumiert werden, insofern Valenzträger die morphologische Form der von ihnen ,regierten' (abhängigen) Elemente bestimmen (,regieren').“ (BUSSMANN 2008:580) Ähnlich wird auch die Rektion in der DudenGrammatik (2009:776) definiert: „Vor allem in Hinblick auf die grammatischen Merkmale spricht man auch von Rektion: Das Wort regiert (verlangt) beispielsweise [...] einen Kasus (= Kasusrektion)“. Hinzuzufügen ist, dass die Rektion einen Teilbereich der Kasuszuweisungsmechanismen darstellt, an denen auch funktionale Kategorien wie die Finitheit beteiligt sind, die für die Zuweisung des als unregiert geltenden Nominativs verantwortlich sind. In neueren generativen Ansätzen werden allerdings Rektion und Nominativzuweisung als Ausprägungen der strukturellen Kongruenzbeziehung betrachtet: Agro für Objektkongruenz (traditionell Rektion), Agrs für Subjektkongruenz (vgl. GREWENDORF 2002:37).

Beim Kasus soll zwischen Kasuswert/-merkmal und Kasusrealisierung unterschieden werden. Verschiedene Kasusmerkmale können nämlich die gleiche Realisierung erfahren, so dass es zu Kasussynkretismen kommt, z. B. realisiert die NP eine Frau in (1) a das Merkmal Nominativ (= Nom) in (1) b Akkusativ (= Akk):

(1) a Eine Frau hat mir Guten Tag gesagt. vs.

(1) b Ich habe eine Frau gesehen.

Umgekehrt kann dasselbe Merkmal morphophonologisch verschiedentlich realisiert werden, wie dies beim Genitiv Singular (= Gen Sg) der Maskulina und Neutra im Deutschen häufig der Fall ist:

(2) Die Form des Stuhls / Stuhles gefällt mir nicht.

Es bestehen zahlreiche Belege für Kasusrealisierungen, die mit den Regeln der deutschen Grammatik im Widerspruch stehen. Dies betrifft v.a. das Gen-s bei Maskulina und Neutra Sg ((3)-(4)) sowie das Dat-n im Plural ((5)-(6)),, ${ }^{2}$ vgl.:

Zusammenhang irrelevant. Der Aufsatz fokussiert oberflächenstrukturelle, morphologisch ausdrückbare Kasusformen, so dass hier von theorieinternen Diskussionen, wie sie etwa von generativen Syntaktikern geführt werden, abgesehen werden kann.

Hierzu vgl. u.a. RowLEY (1987). 
(3) Das Urteil des Bundesverfassungsgericht_zum Anleiheprogramm der EZB ist ein Spiel mit dem Feuer.. ${ }^{3}$

(4) Vom Turm des größten Gotteshaus_der Stadt, der Jakobkirche, die nach dem Vorbild der Lübecker Marienkirche erbaut wurde, hatten wir eine famose Aussicht auf die Stadt am Oderstrom. (Privatmail)

(5) Der vermeintliche General habe „von seinen 25 Milliönchen“ erzählt, die er in den Niederlande_gebunkert haben wollte. (Berliner Zeitung, 5.9.2014, 3)

(6) Und dass den Konzerne_ keine Strafzahlungen wegen überhöhter CO $\square$-Werte drohen.. ${ }^{4}$

Solche Beispiele zeigen, dass es zu normabweichenden Kasusrealisierungen kommt, aber die Kasusmerkmale Gen und Dat entsprechen der Norm und sie werden korrekt am Determinator realisiert. In diesem Beitrag geht es dennoch um abweichende Kasusmerkmale, die sich an entsprechenden Morphemen ablesen lassen, so dass unkorrekte Kasusrealisierungen nicht weiter verfolgt werden.

\section{Kasusgebrauch im Deutschen}

Der folgende Abschnitt konzentriert sich auf Fälle der normwidrigen Rektion im Deutschen, wobei das Belegmaterial im Hinblick auf die regierende Kategorie analysiert wird. Zunächst wird die Verbrektion genauer untersucht, anschließend wird zur präpositionalen Rektion übergegangen.

\subsection{Verbale Rektion}

Kasusveränderungen finden u.a. im adverbalen Bereich statt, wobei die größte Aufmerksamkeit der Verdrängung des Gen durch andere Kasus, v.a. durch den Dat, gewidmet zu sein scheint. Laut gängiger, z.T. populärwissenschaftlicher Meinungen, ist im Deutschen der Gen derjenige Kasus, der am meisten von den Kasusveränderungen betroffen sei. Es ist demnach kein Zufall, dass BASTIAN SICK (2005), sein Buch mit dem Titel Der Dativ ist dem Genitiv sein Tod versah. Abgesehen davon, dass er in diesem Buch dem Gen lediglich etwa sungsgericht-ezb, 8.2.2014, online verfügbar. bar. 
3,5 Seiten widmet, wird unten zu sehen sein, dass auch der Gen den Dat verdrängen kann. Darüber hinaus haben Forschungen ergeben, dass hierfür Differenzierungen vorzunehmen sind, v.a. in Abhängigkeit vom syntaktischen Kontext, in dem der Gen erwartungsgemäß auftreten soll. Und so zeigen u.a. VATER (2007:12) und Di MEOLA (1999, 2000), dass im adverbalen Rektionsbereich der Gen durch konkurrierende Formen des Dat und Akk verdrängt wird, aber auch dass dieser Kasus gerade im präpositionalen Rektionsbereich immer häufiger zum Einsatz kommt. M.E ist es somit verfrüht, den ,Genitivtod' zu beweinen.

Veränderungen in der Rektion gab es schon immer und es bedarf nicht unbedingt einer langen diachronischen Perspektive, um sich dieser Erscheinung bewusst zu werden. Als ein geeignetes Beispiel kann sich erinnern herangezogen werden, bei dem der Gen mit der Präposition (= Präp) an + Akk stark konkurriert. Beispielsweise kommt bei KLAUS MANN (2007) dieses Verb fast ausschließlich mit dem Genitiv. ${ }^{5}$ vor, was nicht unbedingt auf einen individuellen Stil des Autors zurückzuführen sein dürfte. Über ein Treffen von Benedetto Croce mit Thomas Mann und seiner Frau berichtet er folgendermaßen (MANN 2007:640):

(7) Sehr herzlich erinnerte er sich einer Begegnung, die er vor tausend Jahren irgendwo mit euch gehabt [...].

Hierzu ist anzumerken, dass der Text zuerst 1942 in englischer Sprache veröffentlicht wurde und sieben Jahre später als vom Autor übersetzte und erweiterte deutsche Fassung erschien. ${ }^{6}$ Es ist daher nicht auszuschließen, dass sich dieses auf die Grammatik der deutschen Ausgabe ausgewirkt haben mag.

Man kann sogar Fälle - diesmal rezent - feststellen, in denen in ein und demselben Satz beide Formen vorkommen.

Beispiel (8) ist die deutsche Übersetzung eines Ausschnitts der Rede, die Barack Obama im Mai 2016 in Hiroshima hielt:

(8) Die Seelen der Toten sprechen zu uns. Wir erinnern uns aller unschuldig Getöteter, wir erinnern uns an den stummen Schrei der Kinder. ${ }^{7}$

$5 \quad$ Heute gilt der Genitiv nach sich erinnern als gehoben und als veraltet, vgl. http://hypermedia.ids-mannheim.de/evalbu/index.html, 5.10.2016.

$6 \quad$ Vgl. https://de.wikipedia.org/wiki/Der_Wendepunkt, 4.10.2016.

7 http://www.zeit.de/politik/ausland/2016-05/hiroshima-japan-barack-obama-redebesuch-atomwaffen, 27.5.2016, online verfügbar. 
Heute überwiegt nach sich erinnern im unmarkierten Sprachgebrauch an + Akk, mit Gen wird das Verb im gehobenen Stil verwendet (vgl. Duden 2001:483).

Im Folgenden wird auf einzelne Fälle der normwidrigen Verbrektion eingegangen.

\subsubsection{Dativ statt Genitiv}

Heute gibt es nur etwa ein Dutzend Verben (ENGEL et alii 1999:236), die den reinen Gen regieren, u.a. sich annehmen, sich bedienen, bedürfen, sich enthalten, sich entledigen, gedenken und entbehren und selbst nach diesen Verben wird der Kasus häufig durch den Dat ${ }^{8}$ ersetzt, vgl.:

(9) Zimmermann bedient sich Volkstanzmotiven (WDR 3, Musikpassagen, 26.9.2006, 16.25 Uhr, zit. nach Vater 2007:12).

(10) Am 9. Mai gedenkt auch Russland dem Ende des 2. Weltkrieges. (Sat1 Nachrichten, 3.5.2015, 20.00 Uhr)

(11) All diese Leistungen laufen ab, ohne dass wir uns ihnen bewusst werden. (3sat, Lichtblicke f. d. Gehirn, 29.1.2007, 20:25 Uhr, zit. nach VATER 2007:13)

Auf (9) kann die sog. Genitivregel bezogen werden, die nach der DudenGrammatik (vgl. 2009:968) folgendermaßen lautet: „Eine Nominalphrase kann nur dann im Genitiv stehen, wenn sie (i) mindestens ein adjektivisch flektiertes Wort und (ii) mindestens ein Wort mit s- oder $r$-Endung enthält.“ In (9) ist vor Volkstanzmotiven weder das eine noch das andere festzustellen, so dass das Nomen aufgrund der Regel nicht im Gen stehen kann. Ohne die $n$-Endung wäre überhaupt kein Kasus eindeutig ausgedrückt, so dass hier aufgrund der Form Nom, Gen oder Akk in Frage kämen. Allein der Dat ist im Plural am Nomen erkennbar, so dass hier gerade dieser Kasus gewählt wurde. Im Unterschied dazu kann der Gen in (10) (definiter Artikel) und (11) (ihrer statt ihnen) durchaus sichtbar gemacht werden, trotzdem wird hier der Dat realisiert. Nach gedenken ist die Konstruktion mit dem Dat nur in der Schweiz anerkannt, sonst sollte das Verb mit dem Gen verwendet werden (vgl. Duden 2007:351).

\subsubsection{Genitiv statt Dativ}

In (12) und (13) kommen zwei Verben vor, die eindeutig den Dat regieren sollten (vgl. Duden 2001:470, 1717) und laut Norm keine Alternationen zulassen, dennoch wurde hier der Gen verwendet:

Unter Umständen kann man hier Fälle unberücksichtigt lassen, auf die die sog. Genitivregel Anwendung findet (s.o.). 
(12) Das heißt nicht, dass sie ihrer königlichen Pflichten und Privilegien entsagt. (Hannes Gamillscheg, Die Prinzessin und ihr Dichter, Kölner Stadtanzeiger, 22.5.2002, 36, zit. nach VATER 2007:14)

(13) [...] hat sich ganz und gar dieses Komponisten verschrieben. (WDR 3, Mosaik, 2.10.2006, 6:35 Uhr, zit. nach VATER 2007:14)

\subsubsection{Dativ statt Akkusativ}

In (14) betrifft die Kasusalternation die Wendung jmdm. auf den Leim gehen, in der anstelle das Akk der Dat verwendet wird:

(14) Martine Zielkes Gruppe zählt inzwischen mehr als dreißig Frauen, Männer und Ehemänner von Frauen, die einem Betrüger auf dem Leim gegangen sind. (Berliner Zeitung, 5.9.2014, 3)

(14) führt vor Augen, dass nicht einmal die Idiomatik von der Verwendung der ,falschen` Kasus bewahrt ist. Selbst wenn es sich hierbei um einen Tippfehler handelt - vielleicht aus dem Grunde, dass die Buchstaben $m$ und $n$ auf der Tastatur nebeneinander liegen oder dass u.U. niemand den Text sorgfältig Korrektur gelesen hat - ist es ein Fehler mit grammatischen Folgen. In einer Examensarbeit etwa dürfte ein solcher ,Vertipper' als grammatischer Fehler und nicht als Tippfehler klassifiziert werden. Darüber hinaus kommt es verhältnismäßig oft zu solchen Fehlern, so dass man die Behauptung aufstellen dürfte, dass es sich in solchen Fällen nicht bloß um ,Vertipper' handelt.

Wenn es um die Alternative Akk-Dat geht, so werden diese beiden Kasus ebenfalls in ihrer direktiven und lokativen Funktion mitunter verwechselt wie in (15), was nach VATER (2007:14f.) höchstwahrscheinlich stark von der Umgangssprache geprägt und für das Ruhrgebiet typisch ist:

(15) Ebenso schlicht und direkt ist das auf Video festgehaltene „Angstexperiment“ von Helge Janse, der sich mitten in der Nacht in einem Wald begab. (J. Kisters, Raum aus Klängen, Kölner Stadtanzeiger, 20.7.2001, 8, zit. nach Vater 2007:14) Das Verb sich begeben ist eindeutig direktiv zu verstehen, so dass hier der Akk zu erwarten ist, dennoch steht der Dat.

\subsubsection{Genitiv statt Akkusativ}

In (16) liegt noch ein Beispiel für die Ersetzung eines anderen Kasus durch den Gen vor:

(16) Man braucht keines besonderen Schutzes. (Sat1, A. Kallwass, 17.11.2014, 7.27 Uhr) 
Dieser Beleg stammt aus der von Sat1 ausgestrahlten Pseudo-Doku-Show Kallwass greift ein! (früher Zwei bei Kallwass), ist also der gesprochenen Sprache zuzuordnen. Man kann mutmaßen, dass die Moderatorin Kallwass wahrscheinlich zuerst die Verwendung von bedürfen im Sinne hatte, schließlich aber in ihrer Äußerung brauchen bei der Beibehaltung der Grammatik von bedürfen artikulierte. Diese Interpretation liegt insofern nahe, als bedürfen dem Verb brauchen semantisch nahe steht und besonders für Versprecher anfällig ist. Bezeichnend ist auch, dass Kallwass sich nicht korrigierte, obwohl sie im Alltag mit der Sprache arbeitet und ein ausgeprägtes Sprachbewusstsein besitzen dürfte.

\subsubsection{Nominativ statt Akkusativ}

(17) Wir lebten in einem Zustand, den Kriegsenkel rückblickend häufig emotionaler Nebel oder bleierne Schwere nennen. ${ }^{9}$

In (17) liegt ein Objektsprädikativ emotionaler Nebel vor, dessen Kasus mit der Bezugsgröße, in diesem Fall mit dem Relativum den, kongruieren sollte. Dennoch steht die Phrase im Nom. In Richtiges und gutes Deutsch heißt es diesbezüglich: „Nach nennen stehen Akkusativobjekt und Prädikativ im gleichen Kasus: Sie nannte ihn einen dummen Jungen (nicht: ein dummer Junge).“ (Duden 2007:647, vgl. auch Duden-Grammatik 2009:396). In normativer Hinsicht besteht somit kein Zweifel daran, welcher Kasus hier verwendet werden sollte, dennoch hat der Artikelautor einen normwidrigen Kasus gewählt.

Der folgende Abschnitt fokussiert Schwankungen in der präpositionalen Rektion.

\subsection{Präpositionale Rektion}

Nach zahlreichen Präpositionen ist der Kasusgebrauch uneinheitlich, selbst im Standarddeutschen regieren viele Präpositionen zwei Kasus, womit allerdings meist Bedeutungsunterschiede verbunden sind wie im Falle der Wechselpräpositionen, die lokale und direktive Beziehungen ausdrücken: „Einige Präpositionen regieren nur einen Kasus, andere zwei, und die Präposition entlang kann sogar alle drei Kasus [...] regieren. Manche Präpositionen schwanken in ihrer Rektion“ (Duden-Grammatik 2009:606). Und gerade diese in ihrer Rektion „schwankenden“ Präpositionen bilden den Gegenstand des folgenden Abschnitts. 


\subsubsection{Genitiv und / oder Dativ}

Es gibt eine Reihe von Präp wie dank, trotz und wegen, denen selbst in normativen Werken eine gewisse Rektionsfreiheit zwischen Gen und Dat eingeräumt wird (vgl. Duden 2007).

a) dank

Die Präp dank entstand im Zuge der Grammatikalisierung aus Phrasen vom Typ Dank sei seinem Einfluss, was später zu dank seinem Einfluss wurde. Da dank denominalen Charakter besitzt wie z. B. auch kraft, laut, statt, infolge und da denominale Präp vornehmlich mit dem Gen vorkommen, so wird dieser Kasus auch nach dank verwendet. Im Plural überwiegt heute der Gen, der auch standardsprachlich als korrekt gilt. Lediglich bei Nomina ohne Determinatoren im Plural wird in Einklang mit der Genitivregel der Dat gewählt, weil andernfalls der Gen den Kasus nicht erkennen ließe (vgl. Duden 2007:212). Laut Duden ist der Gen hier demnach der Standardfall, es sei denn, im Plural treten artikellose Substantive auf. Diese Regel wird gemeinhin auch befolgt, wie das nachstehende Beispiel verdeutlicht:

(18) Die Großhandelspreise für den Strom sind - auch dank des wachsenden Angebots aus Wind- und Solarparks - auf dem tiefsten Stand seit über einem Jahrzehnt. ${ }^{10}$

Allerdings wurde ein Beleg gefunden, in dem die morphologische Form des Adjektivs auf den Nom / Akk hinweist. Da es aber ein Einzelfall zu sein scheint, ist hier ein Tippfehler nicht ausgeschlossen, obwohl dies nicht mit Sicherheit feststellbar ist:

(19) Nach guten Daten aus Australien, das dank stärkere Nachfrage aus China nach Erz und Kohle wieder einen höheren Handelsbilanzüberschuss ausweist, legte der „Aussie“ kräftig zu.. ${ }^{11}$

b) trotz

Nach Duden (2007:890) steht die Präp trotz heute gewöhnlich mit dem Gen. Der Dat ist älter und mittlerweile seltener als der Gen, was jedoch nicht auf Süddeutschland, Österreich und die Schweiz zutrifft, wo trotz vorzugsweise mit dem Dat verwendet wird (vgl. auch Duden 2001:833). Nach VATER (2007:9) ist „trotz, das vom Verb trotzen abgeleitet ist und ursprünglich den

10 http://www.zeit.de/wirtschaft/unternehmen/2016-04/peabody-energy-kohle-abbau-konzern-usa-insolvenz, 14.4.2016, online verfügbar.

11 http://www.faz.net/aktuell/wirtschaft/konjunktur/weltwirtschaft-das-wahre-gewicht-chinas-12787501.html, 8.2.2014, online verfügbar. 
Dativ regierte (vgl. trotzdem) durch Grammatikalisierung (im Sinne einer Delexikalisierung) zum Genitiv übergegangen“, so dass der Dat die ältere Rektion anzeigt, wovon solche festen Fügungen wie trotz allem und trotz alledem sowie das Adverb trotzdem zeugen. Der Dat erscheint vor allem dann, wenn es um starke Substantive im Plural ohne Determinator geht (s. Genitivregel): trotz Beweisen, trotz Büchern, trotz Atomkraftwerken.

Dat-Gen-Schwankungen sind Ausdruck natürlichen grammatischen Wandels (vgl. Duden-Grammatik 2009:601f.). Beispielsweise verwendet KLAUS MANN (2007) trotz fast immer noch mit Dat:

(20) Auch das Libretto gefiel mir trotz seinen naiven Fehlern. (MANn 2007:600)

Gelegentlich aber auch schon mit Gen (MANN 2007:639):

(21) trotz dieser Übelstände

Interessant, aber schwer einzuordnen sind Fälle, in denen sich in einer Phrase Exponenten beider Kasus feststellen lassen:

(22) trotz diesem Rummels (RTL Aktuell, 19.3.2014, 19.00 Uhr)

Hier zeigt der Determinator den Dat an, die Nomenendung ist aber eindeutig genitivisch. Im weiteren Verlauf dieses Beitrags wird zu sehen sein, dass solche in Bezug auf den Kasus hybriden NPs keine Seltenheit sind, was von einer bestimmten Nachlässigkeit der Sprecher / Sprecherinnen und Schreiber / Schreiberinnen in Bezug auf den Kasusgebrauch in spontanen Äußerungen zeugen kann.

c) entgegen

Im Duden (2007:287) wird auf die verbindliche dativische Rektion von entgegen hingewiesen, aber in der Duden-Grammatik (2009:611, vgl. auch DI MEOLA 1999 und VATER 2007:10) wird der immer häufigere Gebrauch des Gen in der heutigen Sprache konstatiert. Dies kann als Indiz dafür aufgefasst werden, dass sich die Autoren bei ein und demselben Verlag nicht einig bezüglich des Kasusgebrauchs nach dieser Präposition sind.

Nachstehend einige Belege für den Gen nach entgegen:

(23) Die USA hätten sich - entgegen anderer Aussagen vor allem aus Russland auf keine Seite im Ukraine-Konflikt gestellt.. ${ }^{12}$

12 http://www.zeit.de/politik/ausland/2014-02/nuland-reaktion-usa-russland,

7.2.2014, online verfügbar. 
(24) Aber das müssen die Bundesländer verantworten, die entgegen aller Absprachen und deutschen Interessen gegen den Fiskalpakt gestimmt haben. (FAZ, 10.3.2013, 1)

In (24) liegt eine koordinierte Phrase vor, in der nur beim ersten Glied der Kasus eindeutig abzulesen ist; im zweiten Glied der Koordination ist nicht klar, ob Dat oder Gen realisiert ist: deutschen Interessen könnte elliptisch von [entgegen aller] deutschen Interessen (Gen) oder von [entgegen] deutschen Interessen (Dativ) abgeleitet sein.

Drei weitere Belege für den Gen:

(25) Offenbar aber machten die Menschen nun die Erfahrung, dass sich Ungleichheit entgegen aller Aufstiegsversprechen nur schwer überwinden lässt.. ${ }^{13}$

(26) Entgegen früherer Mitteilungen wurden offenbar doch noch keine Wrackteile der ägyptischen Maschine entdeckt. ${ }^{14}$

(27) Das würde entgegen aller internationalen Erfahrungen verlaufen. ${ }^{15}$

Die angeführten Belege sowie die in der Literatur befindlichen Informationen weisen darauf hin, dass nach entgegen eine Tendenz besteht, den Dat zugunsten des Gen aufzugeben.

d) samt / mitsamt

Zu dieser Präp findet man divergierende Auffassungen, und so äußert sich z. B. VATER (2007:10) dahingehend, dass samt heute noch mit Dativ konstruiert, aber sehr oft oder schon überwiegend mit Genitiv gebraucht wird. Derselbe Autor (VATER 2011:245) behauptet in einer vier Jahre später erschienenen Publikation, dass samt heute meist mit Gen gebraucht wird, der Dat wird nicht einmal erwähnt. Nach Duden (2007:784) soll samt dagegen mit dem Dat stehen, auf den Gen wird dort nicht verwiesen. Allerdings finden sich sowohl nach samt als auch nach dem ihm form- und bedeutungsaffinen mitsamt zahlreiche Belege mit Gen-Rektion, vgl.:

http://www.zeit.de/wirtschaft/2016-05/umfrage-deutschland-friedrich-ebert-stiftung-soziale-ungleichheit, 11.5.2016, online verfügbar. kairo-funde-wrackteile, 20.5.2016, online verfügbar. hofer-party, 23.5.2016, online verfügbar. 
(28) Der öffentlich-rechtliche Rundfunk in seiner jetzigen Form soll mitsamt des Rundfunkbeitrags abgeschafft werden. ${ }^{16}$

Es ist nicht auszuschließen, dass solche Formen Ausdruck persönlicher Bevorzugung sind; beispielsweise verwendet RUDI KELLER in seinem Klassiker zum Sprachwandel (2003) samt ausschließlich mit Gen:

(29) Er stieß einen gellenden Schrei der Angst aus, und wiederum war in Sekundenschnelle die Horde samt dieser widerlichen Bonzen wie vom Erdboden verschwunden. (KELLER 2003:39)

(30) [...] auf einer Karte, auf der die Gebäude und sonstigen Einrichtungen samt ihrer Funktionen eingetragen wären, nicht aber die Wege (KeLLER 2003:100).

(31) Die Mikroebene ist die Ebene der Handlungen der beteiligten Individuen samt der relevanten Bedingungen, unter denen sie handeln (KELLER 2003:126).

Die bisher untersuchten Fälle betrafen Schwankungen im Kasusgebrauch, die bereits normiert sind wie bei trotz und dank, oder Kasusgebrauchsfälle, die zwar nicht normgerecht, jedoch häufig anzutreffen sind wie bei entgegen und samt. Im Folgenden werden Belege präsentiert, in denen seltenere (Rektions-) ,Fehler' auftreten; besonders komplex und kaum erklärbar sind Konstruktionen, in denen in ein- und derselben Phrase Morpheme verschiedener Kasus vorkommen oder aber der Kasus nicht identifiziert werden kann.

\subsection{Sonderfälle}

\subsubsection{Inkorrekter Kasus}

(32) In Karstadt steckt sehr viel Handelserfahrung, sehr viel Wissen um die Bedürfnisse unseren Kunden. ${ }^{17}$

In (32) regiert die Bedürfnisse eine NP im Dat, obwohl in adnominaler Stellung der Genitiv erwartbar wäre. Es ist kaum davon auszugehen, dass diese Kasusverwechslung auf einem Tippfehler beruht, weil $r$ und $n$ - im Unterschied etwa zu $n$ und $m$ - auf der Tastatur entfernt voneinander liegen. Plausibler ist hier eine Weglassung der Dat-Präp von vor unseren Kunden, so dass man hier von einem Flüchtigkeitsfehler sprechen könnte. Ist es aber ein ,Vertipper`, so hat er grammatische Folgen, würde allerdings z. B. in einer Examensarbeit wohl als Verschreiber gelten.

http://www.sueddeutsche.de/politik/afd-das-hat-der-afd-parteitag-beschlossen1.2975251, 2.05.2016, online verfügbar. 
In Bezug auf in heißt es in Duden (2001:445): „Die Präposition in kann mit dem Dativ oder mit dem Akkusativ verbunden werden, je nachdem, ob das durch das Verb ausgedrückte Verhalten lagemäßig (Dativ) oder richtungsmäßig (Akkusativ) bestimmt ist“. Es unterliegt keinem Zweifel, dass das in (33) ausgedrückte Verhalten statisch (lagemäßig) bestimmt ist, so dass hier der Dat zu erwarten ist, dennoch wird der Akk realisiert:

(33) Wie weit weg Deutschland von diesem Denken ist, zeigt die in diese Woche in Berlin veröffentlichte Sinus-Jugendstudie. ${ }^{18}$

Im Folgenden werden Beispiele für Koordinationen angeführt, in denen die Konjunkte Exponenten verschiedener Kasus aufweisen.

\subsection{2. ,Koordiniertes Chaos`}

Es liegen zahlreiche Fälle vor, in denen Phrasen mit Exponenten verschiedener Kasus miteinander koordiniert werden. Auf diese Erscheinung ist u.a. in Vater (2007 und 2011) hingewiesen worden. In VATER (2011:251) ist zu lesen, dass Konjunktionen sich in solchen normabweichenden Konstruktionen wie Regenzien verhalten, obwohl sie verbindende Elemente sind und die verbundenen Konstituenten werden von anderen Elementen, z. B. Verben und Präp, regiert. Die koordinierten nominalen Konjunkte müssen denselben Kasus aufweisen. So ist es aber nicht in (34), wo eines der koordinierten Adjektive im Akk und eines im Dat erscheint, obwohl sie beide von unter regiert werden und in diesem Fall im Dat stehen sollten:

(34) In der zweiten Hälfte des 19. Jahrhunderts entwickelte sich jedoch vor allem in der Westukraine, unter polnischen und österreichischem Einfluss, eine ukrainische Identität. ${ }^{19}$

In (35) liegt ebenfalls eine Koordination vor, deren erstes Glied der kommunalen Arbeitgeber vom Nomen Gremien regiert wird und korrekterweise im Gen erscheint. Problematisch ist die Interpretation des zweiten Konjunkts dem Beamtenbundes:

http://www.zeit.de/wirtschaft/2016-04/frankreich-proteste-hollande/seite-2, 28.4.2016, online verfügbar.

19 http://www.zeit.de/politik/ausland/2014-03/russland-ukraine-geschichte, 4.3.2014, online verfügbar. 
(35) Nach den Gremien der kommunalen Arbeitgeber und dem Beamtenbundes dbb stimmte auch die Bundestarifkommission von Ver.di zu. ${ }^{20}$

Mit dem Beamtenbundes sind zumindest zwei Probleme verbunden: Erstens kann aufgrund der Kasusmorpheme nicht festgestellt werden, welcher Kasus überhaupt zugewiesen und realisiert wird, da der Determinator auf den Dat, das Nomen aber auf den Gen hinweist. Dies ist mit dem syntaktischen Grundsatz, dass eine NP einen Kasus zugewiesen bekommt, nicht zu vereinbaren. Das zweite Problem ist eine logische Folge des ersten: Da hier Exponenten des Dativs und Genitivs in einer Phrase vermengt werden, ist nicht zu erschließen, ob das Regens von Beamtenbund die Präp nach (Dat) oder das Nomen Gremien (Gen) ist. Solche evidente Nachlässigkeit beim Kasusgebrauch kann also auch zu Ambiguitäten bei der Sprachverarbeitung führen.

Verständlich, aber inkorrekt ist die dreigliedrige Koordination in (36), in der als Regens die dativregierende Präp von fungiert. Der Dativ ist aber zunächst am ersten Konjunkt der $P K K^{21}$ ausgedrückt, das zweite anderer kurdischextremistischer Splittergruppen steht eindeutig im Genitiv (zwei -er-Endungen im Plural) und das dritte dem „Islamischen Staat“ wieder im Dat:

(36) Da ist die Gefahr von Terroranschlägen, verübt von der PKK oder anderer kurdisch-extremistischer Splittergruppen oder dem „Islamischen Staat“... 22 Auch (37) enthält eine dreigliedrige Koordination, die von aus regiert wird. Die zwei ersten Konstituenten sind artikel- und attributlose Ländernamen. Der erwartbare Dat kann hier folglich nicht sichtbar gemacht werden, wohl aber der Gen in Form der Endung $-s,{ }^{23}$ die jedoch ausbleibt, so dass doch vom Dat auszugehen ist. Das dritte Glied steht aber im Gen:

20 http://www.zeit.de/wirtschaft/2016-04/verdi-lohnerhoehung-oeffentlicher-dienst, 30.4.2016, online verfügbar.

21 Rein äußerlich gesehen könnte der PKK auch den Genitiv repräsentieren, der im Falle der Feminina Sg mit Dativ homophon ist. Da aber die NP direkt der Präposition von folgt, ist hier doch der korrekte Dativ anzunehmen.

22 http://www.zeit.de/politik/ausland/2016-05/turkei-immunitaet-abgeordnete-hdp, 5.5.2016, online verfügbar.

23 Ein zusätzliches Problem besteht darin, dass gerade diese Endung oft aufgegeben wird (s. (3)-(4)), wofür auch zahlreiche Belege angeführt werden könnten. 
(37) Regelmäßig haben damals in den neunziger Jahren Platten aus Schweden, Italien, Belgien und der Niederlande bei uns in den Charts gestanden. ${ }^{24}$

Im letzten dem Deutschen gewidmeten Abschnitt sollen einige Fälle aufgezeigt und diskutiert werden, in denen aufgrund morphologischer Exponenten die Kasusidentifikation erschwert oder gar unmöglich ist.

\subsubsection{Schwierigkeiten bei der Kasusidentifikation}

Ein Fall einer erschwerten Kasusidentifikation wurde bereits anhand der Koordination in (35) diskutiert. Nun sollen einige Belege präsentiert werden, in denen an einer unkoordinierten NP Morpheme verschiedener Kasus ausgedrückt werden.

Präp auf in stolz sein auf verbindet sich mit dem Akk und dieser Kasus sollte auch in (38) realisiert werden:

(38) Er sei besonders stolz auf seine guten Kontakten nach Moskau, so wird es erzählt. ${ }^{25}$

Zwar weist die Konstituente seine guten auf den Akk (oder Nom) hin, aber das Nomen ist eindeutig dativisch markiert. Hier liegt also eine unvereinbare Kasusmarkierung vor.

Weitere Beispiele für ähnliche Erscheinungen sind:

- Determinator im Dat Sg, Nomen im Dat Pl

(39) Kowaltschuk ließ über einen Anwalt mitteilen, sich nicht zu der Sachen äußern zu wollen. ${ }^{26}$

- Determinator im Akk, Adjektiv im beliebigen Kasus, Nomen im Dat (40) Ist die SZ auf die russischen Diensten SWR, FSB, FAPSI reingefallen? ${ }^{27}$

- Determinator im Dat Pl statt Akk nach gegen, Nomen ohne Kasusmarkierung:

24 http://www.faz.net/aktuell/feuilleton/debatten/brexit-robbie-willams-spice-girlsbritische-selbsttaeuschung-14331005.html?printPagedArticle=true\#pageIndex_2, 12.7.2016, online verfügbar.

25 http://panamapapers.sueddeutsche.de/articles/56eff9f22f17ab0f205e636a/, 4.4.2016, online verfügbar.

26 http://panamapapers.sueddeutsche.de/articles/56eff9f22f17ab0f205e636a/, 4.4.2016, online verfügbar.

27 http://www.sueddeutsche.de/medien/panama-papers-konspirologen-und-leibwaechter-des-kapitals-1.2945738, 14.4.2016, online verfügbar. 
(41) Er will nun seine Amtskollegen aus den Bundesländern dazu anhalten, weitere Sondereinheiten gegen den Wohnungseinbrüche zu gründen. ${ }^{28}$

Das nächste Kapitel ist Normverstößen im Kasusgebrauch im Polnischen gewidmet.

\section{Kasusgebrauch im Polnischen}

Polnisch zählt sieben morphologische Kasus: Nom, Gen, Dat, Akk, Instr, Lok und Vok. Kennzeichnend für die letzten beiden ist, dass der Lokativ ausschließlich mit Präpositionen ${ }^{29}$ vorkommt und der Vokativ nur als Anredekasus außerhalb des syntaktischen Verbandes gebraucht wird.

Wie im Deutschen lassen sich auch im Polnischen Veränderungen im Kasusgebrauch feststellen, wobei dieser Prozess im Polnischen systematischer als im Deutschen zu verlaufen scheint, d.h., es ist eine deutliche Tendenz zur Ersetzung des Gen durch den Akk erkennbar. Dies betrifft sowohl den lexikalischen / lexemspezifischen als auch den strukturellen Gen nach der Satznegation. In beiden Fällen hat man es mit der Prädikat-Objekt-Beziehung als dem Bereich zu tun, in dem die meisten syntaktischen Sprachwandelprozesse des Polnischen stattfinden (vgl. PISARKOWA 1984:248).

\subsection{Lexikalischer Genitiv}

Im Polnischen regieren weit mehr Verben den Gen als im Deutschen. Dazu gehören u.a. bronić (verteidigen), oczekiwać (erwarten), pilnować (bewachen), poszukiwać (suchen), strzec (hüten), unikać (vermeiden), używać (benutzen) und andere mehr (vgl. ENGEL et alii 1999:236). Mit manchen der aufgezählten Verben, z. B. nach bronić, oczekiwać, pilnować oder używać wird bereits oft die Akk-Rektion realisiert:

(42) Ciężarówka, [którą]*Akk użył do ataku, była wynajęta. / ,Der LKW, den er für den Anschlag benutzte, war gemietet. ' ${ }^{30}$ (TVN 24, 15.07.2016, 10.02 Uhr)

http://www.sueddeutsche.de/panorama/kriminalitaetsstatistik-zahl-der-wohnungseinbrueche-stark-gestiegen-1.3001715, 21.5.2016, online verfügbar.

Der präpositionslose Lokativ ist gelegentlich nur noch landschaftlich zu hören, z. B. lecie (im Sommer) anstatt des standardsprachlichen w lecie / latem (im Sommer).

Da es hier ausschließlich um NPs im Gen und Akk geht, während andere grammatische Kategorien irrelevant sind, wird auf die deutsche Glossierung der polnischen Beispiele verzichtet. Sie werden dagegen direkt übersetzt und die in Frage 
In (42) steht das von $u \dot{z} y w a c ́$ regierte Relativum im Akk anstatt im normgerechten Gen.

Ebenfalls nach bronić (43) und oczekiwać (44) wird häufig mit Akkusativ konstruiert:

(43) Z narażeniem życia bronili [króla i królową)*akk. (vgl. Markowski 2002:73) / ,Sie verteidigten den König und die Königin, indem sie ihr Leben aufs Spiel setzten.

(44) Chłopak niecierpliwie oczekiwał [dziewczynę]*Akk (vgl. Markowski 2002:556) / ,Der Junge erwartete ungeduldig seine Freundin.

Solche Fälle sind jedoch auf einzelne Lexeme beschränkt und dürften sich nicht in dem Maße auf die Grammatik des Polnischen auswirken wie der immer häufigere Akk nach negierten transitiven Verben.

\subsection{Akkusativ anstelle des Genitivs der Negation}

Im Standardpolnischen wird das direkte Objekt im Akk unter Satznegation zu einer Phrase im Gen. Diese Erscheinung ist außer im Polnischen noch in einigen slawischen Sprachen produktiv, und zwar im Russischen, Belarussischen, Ukrainischen und Slowenischen (vgl. WITKOŚ 1998:232), jedoch wird auch in diesen Sprachen mit verschiedener Häufigkeit der Akk statt des Gen gebraucht. Im Tschechischen ist der Gen in dieser Funktion bereits durch den Akk ersetzt worden (vgl. BUTTLER / KURKOWSKA / SATKIEWICZ 1971:306).

\subsubsection{Darstellung in (normativen) Grammatiken}

In den Grammatiken des Polnischen wird konstatiert, dass Verben, die das direkte Objekt im Akk regieren, eine Genitivrektion aufweisen, wenn sie negiert sind. D.h. der Gen ist hier als Ergebnis eines Rektionswechsels anzusehen (vgl. BUTTLER / KURKOWSKA / SATKIEWICZ 1971:304, FRANKS 1995:202, NAGÓRKO 1998:275, SALONI / ŚWIDZIŃSKI 1998:156f., WITKOŚ 1998:216, JADACKA 2008:152). Nach JADACKA (2008:152) soll dies die einzige ausnahmslose Regel der polnischen Syntax sein. Ähnliches ist auch bei Franks zu lesen (1995:202): „The accusative is never acceptable under sentential negation“. Die grammatische Kraft der Koppelung „Negation $\rightarrow$ Gen“ offenbart sich u.a. darin, dass die Negation den Gen auch über Modalverben hinaus erzwingt, d.h., sie regiert gewissermaßen in Modalverbkomplexe (MVK) hinein (vgl. SALONI / ŚWIDZIŃSKI 1998:157):

stehenden Phrasen werden wie in den polnischen Originalbelegen durch Fettdruck markiert. 
(45) a Musi brać [środki nasenne]Akk. / ,Sie muss Schlafmittel nehmen.

(45) b Nie musi brać [środków nasennych]Gen. / ,Sie muss keine Schlafmittel nehmen.'

Selbst die Grenze der satzwertigen Infinitivkonstruktionen stellt für die Negation keine Rektionsbarriere dar:

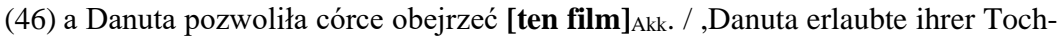
ter, diesen Film zu sehen.

(46) b Danuta nie pozwoliła córce obejrzeć [tego filmu]*Gen. / ,Danuta erlaubte ihrer Tochter nicht, diesen Film zu sehen`

In der mündlichen Sprache werden die genannten Normen jedoch nicht immer befolgt, d.h., es kommt immer häufiger vor, dass der Gen nach negiertem Prädikat durch den normwidrigen Akk ersetzt wird. Selbst im linguistischen Aufsatz von BORSLEY / RIVERO (1994:413, 416) findet man solche dort als grammatisch eingestuften Beispiele wie:

(47) a My byśmy nie czytali [tę książkę]*Akk. / Wir würden dieses Buch nicht lesen.

(47) b Nie będę czytał [tę książkę]*Akk. / Ich werde dieses Buch nicht lesen.‘

JADACKA (2008:152) hebt ähnlich wie PISARKOWA (1984:96) hervor, dass im Polnischen generell die Tendenz herrscht, die Gen- durch die Akk-Rektion zu ersetzen. Dies führt sie unter anderem auf den Einfluss der Massenmedien zurück, die ihrer Meinung nach ,Gegenvorbilder‘ (poln. antywzory) verbreiten. Aufgrund eigener Beobachtungen kann der Autor dieses Aufsatzes dieser Meinung völlig zustimmen.

Die Wahrscheinlichkeit, eine inkorrekte Rektion zu realisieren, nimmt bei jeder semanto-syntaktischen Komplikation zu. Dazu gehören:

a) negiertes Modalverb,

b) Inversion,

c) größere Entfernung zwischen Regens und Dependens, in diesem Fall ist es die Distanz zwischen Prädikat und direktem Objekt (vgl. BuTTLER / KURKOWSKA / SATKIEWICZ 1971:306f.),

d) komplexes Prädikat.

\subsubsection{Beleganalyse}

Im Folgenden wird eine Analyse der Belege im Hinblick auf die oben genannten, den normwidrigen Akk begünstigenden Faktoren durchgeführt, wobei sich die Untersuchung teilweise an den generativ ausgerichteten Aufsatz 
von APTACY (2014) anlehnt. Es wird jeweils versucht festzustellen, welcher Art diese Faktoren sind.

Zunächst werden Äußerungen angeführt, in denen keine strukturellen Begünstigungen des Akk feststellbar sind. Dies wird mit [-str Faktor] gekennzeichnet und bedeutet, dass die Grundabfolge VO realisiert wird, d.h. ohne Linksversetzung des Objekts, und dass kein Modalverb vorhanden ist. Es fällt auf, dass ziemlich viele Konstruktionen im Akk vorkommen, obwohl keine syntaktischsemantischen Komplikationen vorliegen.

\section{Typ 1 [- str. Faktor]}

(48) To nie oznacza [eliminację]*Akk tych dzieci, które się w ten sposób urodziły. (TVP Info, 12.4.2013, 7.22 Uhr) / ,Dies bedeutet keine Eliminierung der Kinder, die auf diese Weise zur Welt gekommen sind.

(49) Nie wpuszczą [rozpędzony pojazd uprzywilejowany]*Akk, bo doszłoby do zderzenia. (Polsat Wydarzenia, 21.5.2013, 19.13 Uhr) / ,Sie lassen keinen rasenden Einsatzwagen herein, denn es würde zu einem Zusammenstoß kommen.

(50) Ona wcale nie deprecjonowała [niskie wynagrodzenia Polaków]*akk W gospodarce. (TVN 24 Bis, 19.7.2016, 9.05 Uhr) / ,Sie hat die Niedriglöhne der Polen in der Wirtschaft überhaupt nicht herabgewürdigt.

(51) Pan Kijowski nie płaci [alimenty]*akk na swoje dzieci. (TVN, 19.1.2016, 19.57 Uhr) / ,Herr Kijowski zahlt keinen Unterhalt für seine Kinder.

Unter Typ 2 werden Äußerungen aufgelistet, in denen Strukturen realisiert werden ([+str. Faktor]), die den Akk wahrscheinlicher machen. Da es mehrere solche Muster gibt, werden innerhalb des Typs 2 Subtypen unterschieden und so sind unter Subtyp 2.1 Konstruktionen mit einem komplexen Prädikat zu finden, allerdings ohne Modalverb, in denen das Objekt rechts vom Verb steht: ${ }^{31}$

Typ 2 [+ str. Faktor]

\section{Subtyp 2.1 [+ str. Faktor, - LV, -MVK]}

(52) Nie jesteśmy w stanie [to]*akk określić (TVP 2 Panorama, 10.11.2011, 18.00 Uhr) / ,Wir sind nicht imstande, das zu bestimmen.

(53) Żydowska hucpa z Jedwabnem nie miała na celu [ustalenie prawdy historycznej]*Akk, [...] (Gazeta Wyborcza, 15.7.2016, 3) / ,Die jüdische Chuzpe bezüglich Jedwabne hatte nicht zum Ziel, die historische Wahrheit zu bestimmen.

31 Die Kürzel werden unter Diagramm (69) erklärt. 
(54) [...] ten sam mówca [...] nie jest w stanie wyartykułować [„ten sam“ wyraz]*akk choćby tylko dwa razy w taki sposób [...] (Grucza 1993:157) / ,Derselbe Sprecher [...] ist nicht imstande, „dasselbe“ Wort zweimal identisch zu artikulieren.'

Subtyp 2.2 umfasst Sätze mit einem Modalverb:

\section{Subtyp 2.2 [+ str. Faktor, - LV, + MVK]}

(55) Gdzie mogą, a gdzie nie powinni układać [te instalacje podziemne]*Akk. (TVN 24, 13.6.2015, 19.13 Uhr) / Wo sie können und wo sie diese Untergrundinstallationen nicht verlegen sollen.

(56) Solidarność nie mogła [ją]*akk pytać o nierówności społeczne. (TVP Info, 9.4.2013, 17.19 Uhr) / ,Die Solidarność konnte sie nicht nach sozialen Ungleichheiten fragen.

(57) Nie można [każdego przedsiębiorcę]*akk traktować jak oszusta. (Tokfm, 24.4.2013, $6.30 \mathrm{Uhr}$ ) / , Man darf nicht jeden Unternehmer wie einen Betrüger behandeln.

(58) Nie możemy ciągle składać [odpowiedzialność]*Akk za nasze niepowodzenia na jednego człowieka. (TVP1, 20.5.2013, 13.32 Uhr) / , Wir dürfen nicht immer für unsere Misserfolge nur einen einzigen Menschen verantwortlich machen.

Beim Subtyp 2.3 hat man es mit Konstruktionen mit linksversetztem Objekt zu tun, d. h., oberflächenstrukturell befindet es sich außerhalb des verbalen Rektionsbereichs, da Verben im Polnischen nach rechts regieren. Allerdings steht das Objekt nicht in der Topik-Position.

\section{Subtyp 2.3 [+ str. Faktor, + LV, - Top]}

(59) Ten komunikat [Naczelną Radę Lekarską]*akk do niczego nie zobowiązuje. (TVP Info, 5.1.2012) / ,Diese Mitteilung verpflichtet die Ärztekammer zu nichts."

(60) Jeśli człowiek [coś]*Akk nie kupuje w aptece, to się boi. (Tokfm, 9.6.2015, 10.02 Uhr) / ,Wenn man etwas nicht in der Apotheke kauft, hat man Angst.

(61) Jak państwo [te obietnice]*Akk nie przyjęli, to się zaczęło straszenie. (TVP Info, 21.7.2015, 17.53 Uhr) / ,Als Sie diese Versprechen nicht angenommen haben, hat die Angstmache angefangen.'

Subtyp 2.4 unterscheidet sich vom Subtyp 2.3 dadurch, dass die linksversetzte Konstituente sich in der Topik-Position befindet. In 2.4.1 ist kein Modalverb vorhanden, in 2.4.2 dagegen tauchen Modalverben auf. 
Subtyp 2.4 [+ str. Faktor, + LV, + Top]

Subtyp 2.4.1 [+ str. Faktor, + LV, + Top, - MVK]

(62) [To miasto]*Akk ten rozejm nie obejmował. (TVN 24 Bis, 27.2.2015, 23.53 Uhr) / ,Diese Stadt umfasste der Waffenstillstand nicht. ‘

(63) [Sankcje na wołowinę]*Akk nie zdjęła z nas Ukraina (TVN 24, 5.5.14, 00.19 Uhr) / ,Sanktionen für Rindfleisch hat die Ukraine für uns nicht aufgehoben.

(64) [Taki precedens]*akk Polska sobie nie życzy. (TVN Fakty, 24.8.2014, 19.21 Uhr) / ,So einen Präzedenzfall wünscht sich Polen nicht.

(65) [Nasze doświadczenia]*Akk prosimy nie przekładać na Platformę Obywatelską. (TVN 24, 7.9.2014, 11.43 Uhr) / ,Unsere Erfahrungen bitten wir nicht auf die Bürgerplattform zu übertragen.

\section{Subtyp 2.4.2 [+ str. Faktor, + LV, + Top, + MVK]}

(66) Czy [tę instytucję referendum]*akk nie należałoby odświeżyć? (TVN 24, 3.5.2013, 19.45 Uhr) / ,Sollte man die Institution der Volksabstimmung nicht auffrischen?‘

(67) [Wynik]*Akk nie można uznać za słaby. (Tokfm, 14.8.2013, 7.24 Uhr) / ,Das Ergebnis kann man nicht für schwach halten. ‘

(68) [Film]*Akk nie można uznać za dowód przed sądem. (TVP1, 15.9.2014, 19.38 Uhr) / ,Einen Film kann man nicht als Beweis vor Gericht anerkennen.

Eine Typologie der dargestellten Strukturen soll das folgende, binär konzipierte Diagramm veranschaulichen:

(69) Typologievorschlag

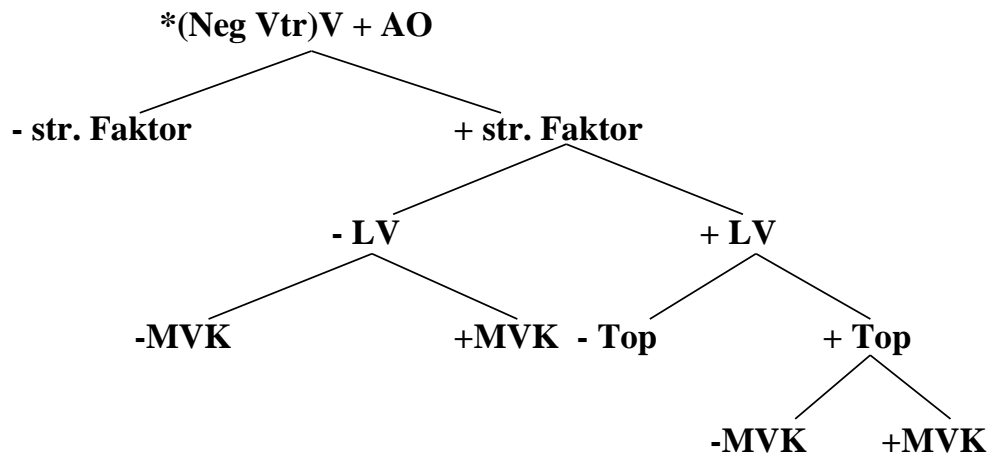


Legende:

*(Neg $\left.\mathrm{V}_{\mathrm{tr}}\right)_{\mathrm{V}}+\mathrm{AO}$ - ungrammatische Konstruktion mit negiertem transitivem Verb,

\pm str. Faktor - Faktor, der den Akk begünstigt, (nicht) vorhanden

$\pm \mathrm{LV}$ - Linksversetzung des Objekts (nicht) vorhanden,

\pm MVK - Modalverbkomplex (nicht) vorhanden,

\pm Top - Objekt (nicht) in der Topik-Position.

\section{Fazit}

Aus der für die beiden Sprachen durchgeführten Analyse ergibt sich folgendes Bild: Die präsentierten Belege führen vor Augen, dass im Deutschen starke Veränderungen im traditionellen Kasusgebrauch stattfinden, was von einer zunehmenden Unsicherheit der Sprecher und Sprecherinnen / Schreiber und Schreiberinnen des Deutschen im Gebrauch dieser grammatischen Kategorie zeugt. Möglicherweise ist das als Hinweis darauf zu deuten, dass Deutsch als indogermanische Sprache im schrittweisen Übergang vom synthetischen zum analytischen Sprachtyp begriffen ist. Das Ergebnis dieses Prozesses könnte langfristig sein, dass diese Sprache in Zukunft die morphologische Kasusmarkierung weitgehend aufgibt, was in anderen germanischen Sprachen bereits erfolgte; Deutsch ist nämlich heute neben Isländisch in Bezug auf die Kasusmorphologie die komplexeste Sprache. Über die Ursachen dieser Entwicklung kann nur gemutmaßt werden, Einflüsse des Englischen und der Sprachmischungen wie ,Kiezdeutsch“ werden in Erwägung gezogen, gelten aber als (noch) nicht bewiesen (vgl. VATER 2007:41, Anm. 44).

Das Polnische unterscheidet sich in dieser Hinsicht vom Deutschen dadurch, dass die Kasusveränderungen systematischeren Charakter besitzen: Sowohl der lexikalische als auch der strukturelle Genitiv werden durch den Akkusativ verdrängt. Äußerungen mit normwidrigem Akk kommen vor allem in der gesprochenen Sprache und in den Massenmedien vor, so dass vom großen Einfluss auf die Sprachgewohnheiten der Sprachbenutzer/innen auszugehen ist. Künftig kann es zum Schwund des Genitivs unter Satznegation wie im Tschechischen kommen. ${ }^{32}$ Dafür kann es zweierlei Gründe geben: Erstens führt der

32 Kotin (persönliche Mitteilung) vertritt die These, dass es zur Aufgabe des Genitivs unter Satznegation kommen muss. 
Akk zur Redundanzvermeidung, da der strukturelle Gen semantisch nicht interpretiert wird, so dass aus Sicht der Optimalität des Sprachsystems dieser Kasus unter Satznegation völlig überflüssig ist. Zweitens dürfte auch die Allgegenwart des Englischen, in dem die Kasusmarkierung schon fast vollständig aufgegeben ist, diese Entwicklung fördern.

\section{Literatur}

Aptacy, JarosŁaw (2014): Phrasenstruktur und Genitiv der Negation im Polnischen - Anzeichen eines Sprachwandels? In: BARTOSZEWICZ, IwONA / SzCZĘK, JOANNA / TwOreK, Artur (eds.): Phrasenstrukturen und -interpretationen im Gebrauch I. Wrocław / Dresden (=Linguistische Treffen in Wrocław 10), 101-112.

Borsley, Robert D. / Rivero, MAria L. (1994): Clitic Auxiliaries and Incorporation in Polish. In: Natural Language \& Linguistic Theory 12/3:373-422.

Bussmann, Hadumod (ed.) ( $\left.{ }^{4} 2008\right)$ : Lexikon der Sprachwissenschaft. Stuttgart.

Buttler, Danuta / Kurkowska, Halina / SATKiewicz, Halina (eds.) (1971): Kultura języka polskiego [Kultur der polnischen Sprache]. Warszawa.

Di Meola, Claudio (1999): Entgegen, nahe, entsprechend und gemäß. Dativpräpositionen mit Genitivrektion. In: Zeitschrift für Germanistische Linguistik 27:344-351.

Di Meola, Claudio (2000): Die Grammatikalisierung deutscher Präpositionen. Tübingen (=SDG 62).

DUden ( $\left.{ }^{4} 2001\right):$ Deutsches Universalwörterbuch. Mannheim.

DUDEN (2001): Richtiges und gutes Deutsch. Wörterbuch der sprachlichen Zweifelsfälle. Mannheim: (Duden Band 9).

DUDEN ( $\left.{ }^{6} 2007\right)$ : Richtiges und gutes Deutsch. Wörterbuch der sprachlichen Zweifelsfälle. Mannheim (Duden Band 9).

Duden ( $\left.{ }^{8} 2009\right)$ : Duden. Die Grammatik. Mannheim.

ENGEL, UlRICH et alii (1999): Deutsch-polnische kontrastive Grammatik. Heidelberg.

Franks, SteVen (1995): Parameters of Slavic Morphosyntax. New York/Oxford (=Oxford Studies in Comparative Syntax).

Grewendorf, GÜNTHER (2002): Minimalistische Syntax. Tübingen / Basel (=UTB 2313).

GRUCZA, FRANCISZEK (1993): Język, ludzkie właściwości językowe, językowa zdolność ludzi [Die Sprache, menschliche Spracheigenschaften, die Sprachfähigkeit der Menschen]. In: PIONTEK, JANUSZ/WIERCIŃSKA, AlinA (eds.): Człowiek w perspektywie ujęć biokulturowych [Der Mensch in biokultureller Perspektive]. Poznań, 151-174.

JADACKA, HANNA (2008): Kultura języka polskiego. Fleksja, słowotwórstwo, składnia [Kultur der polnischen Sprache. Flexion, Wortbildung, Syntax]. Warszawa. 
ManN, KLAus ( $\left({ }^{19} 2007\right)$ : Der Wendepunkt. Ein Lebensbericht. Mit einem Nachwort von Frido Mann. Reinbek bei Hamburg.

MARKowSKI, ANDRZEJ (ed.) (2002): Nowy stownik poprawnej polszczyzny [Neues Wörterbuch für korrektes Polnisch]. Warszawa.

Pisarkowa, Krystyna (1984): Historia Składni Jezyka Polskiego [Geschichte der polnischen Syntax]. Wrocław / Warszawa / Kraków / Gdańsk/ Łódź.

RoWley, Anthony (1987): Zum Genitiv des ganz besonderen Typ. In: Muttersprache 89:58-68.

SALONI, ZygmUnt / ŚWIDZIŃSKI, MAREK ( $\left.{ }^{4} 1998\right)$ : Sktadnia wspótczesnego języka polskiego [Syntax des Gegenwartspolnischen]. Warszawa.

Sick, BASTIAN ( $\left.{ }^{19} 2005\right)$ : Der Dativ ist dem Genitiv sein Tod. Ein Wegweiser durch den Irrgarten der deutschen Sprache. Köln / Hamburg.

VATER, Heinz (2007): Kasusgebrauch im gegenwärtigen Deutschen. In: Estudios Filológicos Alemanes 14:9-46.

VATER, HeINZ (2011): Kasusveränderungen im gegenwärtigen Deutschen. In: KotIN, Michail L., Kotorova, Elizaveta G. (eds.): Geschichte und Typologie der Sprachsysteme. History and Typology of Language Systems. Heidelberg, 245-256.

WitKoś, JACEK (1998): The Syntax of Clitics. Steps towards a Minimalist Account. Poznań 